\title{
A NON-NUMERIC APPROACH TO UNCERTAIN REASONING
}

\author{
Y.Y. Yao \\ Department of Mathematical Sciences, Lakehead University \\ Thunder Bay, Ontario, Canada P7B 5E1 \\ S.K.M. Wong \\ Department of Computer Science, University of Regina \\ Regina, Saskatchewan, Canada S4S 0A2 \\ L.S. Wang \\ Department of Electrical and Computer Engineering \\ McMaster University \\ Hamilton, Ontario, Canada L8S 4K1
}

\begin{abstract}
This paper presents a non-numeric approach to uncertain reasoning by extending the incidence calculus. In parallel to the well known fuzzy, belief/plausibility, probability, and necessity/possibility measures, the corresponding classes of non-numeric functions are examined. A method of constructing non-numeric functions is discussed using the notion of compatibility relations. Non-numeric functions are used to interpret uncertain reasoning by providing possible-worlds semantics for both qualitative and quantitative methods.

INDEX TERMS: Belief/plausibility, fuzzy measure, incidence calculus, interval structure, necessity/possibility, probability, non-numeric functions, rough sets, uncertain reasoning.
\end{abstract}




\section{INTRODUCTION}

The study of uncertainty management has produced a variety of mechanisms for automated reasoning. These mechanisms can be broadly divided into two categories: the numeric and non-numeric (or the qualitative and quantitative) approaches, which capture different but complementary aspects of uncertain knowledge [Bhatnagar and Kanal, 1986]. Typical examples of the numeric approach are probabilistic logic [Nilsson, 1986; Rescher, 1969], fuzzy logic [Dubois and Prade, 1988], belief measures [Nguyen, 1978; Shafer, 1976, 1987; Smets, 1988] and the calculus of certainty factors [Shortliffe, 1976]. The non-numeric approach includes, for instance, propositional logic, incidence calculus [Bundy, 1985, 1986] and rough sets [Pawlak, 1982, 1984].

The non-numeric approach may be used to provide interpretations of the numeric approach as in a possible-worlds semantics analysis. This analysis consists of a propositional language, a set of possible worlds, and a valuation function that maps each proposition to a truth value with respect to a particular possible world [Ruspini, 1991a]. A proposition is characterized by a subset of possible worlds in which the proposition is true. The logical operations are interpreted in terms of settheoretic operations. Within this framework, Bundy [1985, 1986] used an incidence mapping, a non-numeric function, to provide possible-worlds semantics for propositional and probabilistic logics. Similar methods were also studied by many authors [Fagin and Halpern, 1991; Nilsson, 1986; Ruspini, 1991a]. Recently, Wong, Wang and Yao [1992a, 1992b] examined a class of non-numeric functions called interval structure or non-numeric belief. Interval structure provides a common framework that unifies the notions of lower/upper incidence sets [Bundy, 1985] and rough sets [Pawlak, 1982, 1984]. The non-numeric approach is also useful in symbolic computation, machine learning, classification and clustering [Bhatnagar and Kanal, 1986; Jean-Louis, 1991; Luzeaux, 1991; Orlowska, 1985; Pawlak, 1984].

In each of the above studies, only a subclass of non-numeric functions is considered. It is perhaps useful to have a more complete analysis on different classes of nonnumeric functions and their applications in uncertain reasoning. The main objective of this paper is to carry out such an investigation, with emphasis on the characteriza- 
tion, classification, construction and interpretation of various non-numeric functions. In particular, with respect to the well known fuzzy, belief/plausibility, probability, and necessity/possibility measures, we will identify and examine the properties of their corresponding classes of non-numeric functions. We will also discuss a method for constructing non-numeric functions using the notion of compatibility relations [Shafer, 1987]. In our approach, non-numeric functions can be used to provide the possible-worlds semantics for uncertain reasoning. Our analysis extends the existing methods by using a partial, rather than a total, valuation function.

Based on modal logic, Resconi, Klir and Clair [1992], Resconi et. al. [1993], and Harmanec, Klir and Resconi [1993] established a unifying framework within which various uncertainty theories can be formalized, compared and organized hierarchically. The present study may be considered as another and complementary investigation along the same line. The method used by us to relate non-numeric functions and numeric measures is essentially similar to their definition of numeric measures using modal operators.

\section{NON-NUMERIC REPRESENTATION OF UN- CERTAINTY}

Suppose $\Theta=\left\{\theta_{1}, \ldots, \theta_{n}\right\}$ is a finite set of all possible answers to a given question based on one's knowledge, and only one of these answers is correct. This set $\Theta$ is referred to as the frame of discernment or simply the frame defined by the question [Shafer, 1976]. Any subset $A \subseteq \Theta$ is regarded as a proposition representing the fact that the correct answer to the question lies in $A$. The power set $2^{\Theta}$ of $\Theta$ denotes the set of all propositions discerned by the frame $\Theta$. In a situation with incomplete or vague information, it is not possible to say with certainty which subset of $\Theta$ contains the correct answer. However, based on the available information, it is possible to express one's belief in different propositions. A mapping from $2^{\Theta}$ to $[0,1]$ is used to measure such uncertainty. Many numeric measures have been widely used, such as the fuzzy, belief/plausibility, probability, and necessity/possibility measures. These measures differ in the axioms that define them. 
Alternatively, one may represent uncertainty by a non-numeric or symbolic function. Instead of using a numeric value, one may associate a set of points or possible worlds to a proposition [Bundy, 1985, 1986]. Formally, a non-numeric function can be defined as a mapping $i$ from $2^{\Theta}$ to the power set $2^{W}$ formed by a distinct but related frame $W$. The elements in the set $W$ may have different interpretations depending on the context in which a non-numeric function is used. A non-numeric function is defined by certain axioms. In this study, the following list of axioms is considered: for $A, B \subseteq \Theta$,

$(\mathrm{A} 0) \quad \emptyset \subseteq i(A) \subseteq W$,

(A1) $\quad i(\emptyset)=\emptyset$,

(A2) $i(\Theta)=W$,

(A3) $\quad A \supseteq B \Longrightarrow i(A) \supseteq i(B)$,

(A4) $\quad i(A \cap B)=i(A) \cap i(B)$,

(A5) $\quad i(A \cup B)=i(A) \cup i(B)$,

(A6) $\quad i(A) \supseteq i(B)$ or $i(B) \supseteq i(A)$,

(A7) $\quad i(A \cap B) \subseteq i(A) \cap i(B)$,

(A8) $\quad i(A \cup B) \supseteq i(A) \cup i(B)$,

(A9) $\quad i\left(A^{c}\right)=W-i(A)$.

Axiom (A1) and (A2) are assumed for the purpose of normalization. Axiom (A3) requires that a non-numeric function is monotonic with respect to the set inclusion ?. Axioms (A4), (A5) and (A9) state the truth-functionality of the operations $\cap, \cup$ and ${ }^{c}$, such that the value of a composite formula can be computed from the values of its subexpressions. Axiom (A6) states that the values of any two subsets of $\Theta$ are comparable under the set-inclusion relation. Axioms (A7) and (A8) are weaker versions of axioms (A4) and (A5).

Axioms (A1)-(A9) are not a set of independent axioms. Some of the important relationships between these axioms are summarized below:

(I1) $\quad(\mathrm{A} 4) \Longrightarrow(\mathrm{A} 8)$, 


$$
\begin{aligned}
& (\mathrm{A} 5) \Longrightarrow(\mathrm{A} 7) \\
& (\mathrm{A} 7) \Longleftrightarrow(\mathrm{A} 8) \Longleftrightarrow(\mathrm{A} 3), \\
& {[(\mathrm{A} 1),(\mathrm{A} 2),(\mathrm{A} 4),(\mathrm{A} 5)] \Longrightarrow(\mathrm{A} 9)} \\
& {[(\mathrm{A} 4),(\mathrm{A} 9)] \Longleftrightarrow[(\mathrm{A} 5),(\mathrm{A} 9)]} \\
& {[(\mathrm{A} 4),(\mathrm{A} 9)] \Longrightarrow[(\mathrm{A} 1),(\mathrm{A} 2)]}
\end{aligned}
$$

Additional relationships can be derived. For example, from (I5) and (I6) one can infer that $[(\mathrm{A} 5),(\mathrm{A} 9)] \Longrightarrow[(\mathrm{A} 1),(\mathrm{A} 2)]$.

To examine the connection between numeric measures and non-numeric functions, we draw a correspondence between axioms (A1)-(A9) and those axioms that characterize numeric measures. Since the values of a non-numeric function are subsets of a set $W$, it is natural to adopt cardinalities of sets to construct a numeric measure. Corresponding to a non-numeric function $i: 2^{\Theta} \longrightarrow 2^{W}$, we can define a numeric measure $f: 2^{\Theta} \longrightarrow[0,1]$ by:

$$
f(A)=\frac{|i(A)|}{|W|}
$$

where $|\cdot|$ denotes the cardinality of a set. Using this normalized measure $f$, one can analyzing the correspondence between non-numeric axioms (A1)-(A9) and their numeric counterparts, and classify non-numeric functions in the same manner that numeric measures are classified. It is interesting to note that Resconi, Klir and Clair [1992], and Resconi et. al. [1993] used a similar numeric function in their study of various types of numeric measures.

\subsection{Fuzzy Measure}

Consider a non-numeric function $i: 2^{\Theta} \longrightarrow 2^{W}$ characterized by three independent axioms (A1), (A2) and (A3). According to property (I3), axiom (A3) can be replaced by either (A7) or (A8). In this case, a numeric measure defined by equation (1) is a fuzzy measure satisfying the following axioms [Sugeno, 1974]:

$$
\begin{aligned}
& f(\emptyset)=0, \\
& f(\Theta)=1, \\
& A \supseteq B \Longrightarrow f(A) \geq f(B) .
\end{aligned}
$$


Axioms (F1) and (F2) are normalization conditions. They are inferred from the nonnumeric axioms (A1) and (A2). Similar to axiom (A3), axiom (F3) states that a fuzzy measure is monotonic with respect to set inclusion. Clearly, the non-numeric axioms (A1)-(A3) correspond to the numeric axioms (F1)-(F3). We may therefore call a non-numeric function satisfying axioms (A1)-(A3) a non-numeric fuzzy measure.

\subsection{Belief and Plausibility}

A non-numeric fuzzy measure is not truth-functional with respect to both operations $\cap$ and $\cup$. Suppose the truth-functionality of $\cap$ holds. This defines an important class of non-numeric functions satisfying axioms (A1), (A2) and (A4). From property (I1), we obtain $i(A \cup B) \supseteq i(A) \cup i(B)$. Such a function is therefore not necessarily truthfunctional with respect to $\cup$. On the other hand, its dual defined by:

$$
i^{\prime}(A)=W-i\left(A^{c}\right)
$$

is truth-functional with respect to $U$, but not necessarily with respect to $\cap$. Note that $i^{\prime}$ can be equivalently defined by axioms (A1), (A2) and (A5). Axioms (A1) and (A4) imply that, for any proposition $A, i(A) \cap i\left(A^{c}\right)=i(\emptyset)=\emptyset$, and in turn, $i(A) \cap i^{\prime}(A)=i(A) \cap\left(W-i\left(A^{c}\right)\right)=i(A)$. Thus, $i(A) \subseteq i^{\prime}(A)$. The two subsets $i(A)$ and $i^{\prime}(A)$ form an interval set $\left[i(A), i^{\prime}(A)\right]$, representing the qualitative uncertainty of proposition $A$ [Yao, 1993].

The numeric measure $f$ corresponding to such a non-numeric function $i$ is a belief measure defined by axioms (F1), (F2) and the superadditivity axiom:

(F4) For every positive integer $n$ and every collection $A_{1}, \ldots, A_{n} \subseteq \Theta$,

$$
\begin{aligned}
f\left(A_{1} \cup A_{2} \ldots \cup A_{n}\right) & \geq \sum_{i} f\left(A_{i}\right)-\sum_{i<j} f\left(A_{i} \cap A_{j}\right) \\
& \pm \ldots+(-1)^{n+1} f\left(A_{1} \cap A_{2} \ldots \cap A_{n}\right) .
\end{aligned}
$$

The counterpart of the non-numeric function $i^{\prime}$ is the dual of the belief measure $f$ defined by:

$$
f^{\prime}(A)=1-f\left(A^{c}\right)
$$


It is called a plausibility measure, and can be equivalently defined by axioms (F1), (F2) and the subadditivity axiom:

(F5) For every positive integer $n$ and every collection $A_{1}, \ldots, A_{n} \subseteq \Theta$,

$$
\begin{aligned}
f^{\prime}\left(A_{1} \cap A_{2} \ldots \cap A_{n}\right) & \leq \sum_{i} f^{\prime}\left(A_{i}\right)-\sum_{i<j} f^{\prime}\left(A_{i} \cup A_{j}\right) \\
& \pm \ldots+(-1)^{n+1} f^{\prime}\left(A_{1} \cup A_{2} \ldots \cup A_{n}\right)
\end{aligned}
$$

For any proposition, the belief $f(A)$ is less than or equal to the plausibility $f^{\prime}(A)$. The interval $\left[f(A), f^{\prime}(A)\right]$ represents the quantitative uncertainty of proposition $A$. A belief function may be interpreted in terms of a mutli-valued mapping between two frames or using the notion of random sets [Dempster, 1967; Nguyen, 1978].

The superadditivity axiom (F4) is implied by the non-numeric axiom (A4), and the subadditivity axiom (F5) by axiom (A5). Axioms (A1), (A2) and (A4) define a non-numeric belief function, while axioms (A1), (A2) and (A5) define a non-numeric plausibility function. This notion of non-numeric belief and plausibility functions was introduced by Wong, Wang and Yao [1992b]. It has also been studied within the frameworks of incidence calculus [Bundy, 1985, 1986], rough sets [Pawlak, 1982, 1984], and common knowledge [Jean-Louis, 1991].

In the numeric framework, a belief measure can be equivalently defined by another mapping, $m: 2^{\Theta} \longrightarrow[0,1]$, which is called a basic probability assignment satisfying:

$$
\begin{aligned}
& m(\emptyset)=0, \\
& \sum_{A \subseteq \Theta} m(A)=1 .
\end{aligned}
$$

A subset $A \subseteq \Theta$ with $m(A)>0$ is called a focal element. Using the basic probability assignment, the belief $f(A)$ and plausibility $f^{\prime}(A)$ can be expressed as:

$$
\begin{aligned}
& f(A)=\sum_{B \subseteq A} m(B), \\
& f^{\prime}(A)=\sum_{B \cap A \neq \emptyset} m(B) .
\end{aligned}
$$

By the Möbius inversion, from a belief measure one can construct the corresponding 
basic probability assignment [Shafer, 1976]:

$$
m(A)=\sum_{B \subseteq A}(-1)^{|A-B|} f(B) .
$$

In the non-numeric approach, a non-numeric belief function $i$ can be equivalently defined by a basic set assignment, $j: 2^{\Theta} \longrightarrow 2^{W}$, satisfying the following axioms: for any $A, B \subseteq \Theta$,

$$
\begin{aligned}
& j(\emptyset)=\emptyset, \\
& \bigcup_{A \subseteq \Theta} j(A)=W, \\
& A \neq B \Longrightarrow[j(A) \cap j(B)=\emptyset] .
\end{aligned}
$$

A subset $A \subseteq \Theta$ with $j(A) \neq \emptyset$ is called a focal set [Wong, Wang and Yao, 1992b]. Based on the basic set assignment $j$, the non-numeric belief and plausibility can be expressed as:

$$
\begin{aligned}
& i(A)=\bigcup_{B \subseteq A} j(B), \\
& i^{\prime}(A)=\bigcup_{A \cap B \neq \emptyset} j(B) .
\end{aligned}
$$

Conversely, from a non-numeric belief function $i$, the basic set assignment $j$ can be constructed by:

$$
j(A)=i(A)-\bigcup_{B \subset A} i(B)
$$

Furthermore, from the numeric measure $f$ defined by equation (1), we obtain:

$$
m(A)=\frac{|j(A)|}{|W|} .
$$

It is evident that the basic set assignment of a non-numeric belief function corresponds to the basic probability assignment of a numeric belief measure.

\subsection{Probability}

If a non-numeric function is truth-functional with respect to both operations $\cap$ and $\cup$, we call such a mapping an incidence mapping [Bundy, 1985, 1986] which satisfies axioms (A1), (A2), (A4) and (A5). Its corresponding numeric measure $f$ is a 
probability measure defined by (F1), (F2) and the additivity axiom:

$$
A \cap B=\emptyset \Longrightarrow[f(A \cup B)=f(A)+f(B)] .
$$

This additivity axiom corresponds to the non-numeric axioms (A4) and (A5). An incidence mapping can be viewed a non-numeric probability function.

A probability measure is self-dual and can be considered as a special belief measure. The focal elements of a probability measure are singleton sets. Conversely, a pair of belief and plausibility measures can be regarded as the bounds of a set of probability measures [Fagin and Halpern, 1991]. A probability measure $f$ is said to be bounded by a belief measure $\underline{f}$ and the corresponding plausibility measure $\bar{f}$, if $\underline{f}(A) \leq f(A) \leq \bar{f}(A)$ for every $A \subseteq \Theta$. Let $\mathcal{P}$ denote the set of all probability measures bounded by $\underline{f}$ and $\bar{f}$. The following theorem given by Dempster [1967] states the inherent relationship between belief and probability measures.

THEOREM 1 Let $\underline{f}$ be a belief measure on $\Theta$ and $\bar{f}$ be the corresponding plausibility measure. Then for all $A \subseteq \Theta$,

$$
\begin{aligned}
& \underline{f}(A)=\inf _{f \in \mathcal{P}} f(A), \\
& \bar{f}(A)=\sup _{f \in \mathcal{P}} f(A),
\end{aligned}
$$

where $\inf _{f \in \mathcal{P}} f(A)$ and $\sup _{f \in \mathcal{P}} f(A)$ are the lower and the upper envelopes of $\mathcal{P}$.

Similarly, a non-numeric probability function is self-dual, namely, $i(A)=i^{\prime}(A)$ for all $A \subseteq \Theta$. It can be viewed as a special kind of non-numeric belief function. Any focal set of a non-numeric probability function is a singleton set. The basic set assignment is in fact a non-numeric probability distribution. A pair of non-numeric belief and plausibility functions $\underline{i}$ and $\bar{i}$ form the bounds of a set of non-numeric probability functions. A non-numeric probability function $i$ is said to be bounded by $\underline{i}$ and $\bar{i}$, if $\underline{i}(A) \subseteq i(A) \subseteq \bar{i}(A)$ for every $A \subseteq \Theta$. Let $\mathcal{I}$ denote the set of all nonnumeric probability functions bounded by a pair of non-numeric belief and plausibility functions $\underline{i}$ and $\bar{i}$. The lower envelope $\inf _{i \in \mathcal{I}} i(A)$ of $\mathcal{I}$ is defined as the subset of $W$ such that $\inf _{i \in \mathcal{I}} i(A) \subseteq i(A)$ for all $i \in \mathcal{I}$, and that for any $X \subseteq W$, if $X \subseteq i(A)$ for 
all $i \in \mathcal{I}$, then $X \subseteq \inf _{i \in \mathcal{I}} i(A)$. The upper envelope $\sup _{i \in \mathcal{I}} i(A)$ of $\mathcal{I}$ is defined as the subset of $W$ such that $i(A) \subseteq \sup _{i \in \mathcal{I}} i(A)$ for all $i \in \mathcal{I}$, and that for any $X \subseteq W$, if $i(A) \subseteq X$ for all $i \in \mathcal{I}$, then $\sup _{i \in \mathcal{I}} i(A) \subseteq X$. In fact, the lower and upper envelopes $\inf _{i \in \mathcal{I}} i(A)$ and $\sup _{i \in \mathcal{I}} i(A)$ can be computed by the following formulas:

$$
\begin{aligned}
& \inf _{i \in \mathcal{I}} i(A)=\bigcap_{i \in \mathcal{I}} i(A), \\
& \sup _{i \in \mathcal{I}} i(A)=\bigcup_{i \in \mathcal{I}} i(A) .
\end{aligned}
$$

The counterpart of Theorem 1 is given below.

THEOREM 2 Let $\underline{i}$ and $\bar{i}$ be a pair of non-numeric belief and plausibility functions. Then for all $A \subseteq \Theta$,

$$
\begin{aligned}
& \underline{i}(A)=\inf _{i \in \mathcal{I}} i(A), \\
& \bar{i}(A)=\sup _{i \in \mathcal{I}} i(A) .
\end{aligned}
$$

That is, the non-numeric belief function $\underline{i}$ and the plausibility function $\bar{i}$ are the lower and the upper envelopes of $\mathcal{I}$.

Proof. By definition, for all $i \in \mathcal{I}, \underline{i}(A) \subseteq i(A) \subseteq \bar{i}(A)$. Therefore, we only need to prove that $\underline{i}(A)$ and $\bar{i}(A)$ are achievable by some non-numeric probability bounded by $\underline{i}$ and $\bar{i}$. This can be done by constructing, for each proposition $A$, a non-numeric probability $i \in \mathcal{I}$ such that $i(A)=\underline{i}(A)$. For a non-numeric belief $\underline{i}$, there is a basic set assignment, $j: 2^{\Theta} \longrightarrow 2^{W}$, satisfying axioms (S1)-(S3). With respect to $A \subseteq \Theta$, for each $C \subseteq \Theta$ with $j(C) \neq \emptyset$, one can construct a mapping $j_{C}: C \longrightarrow 2^{j(C)}$ satisfying the following conditions:

$$
\begin{aligned}
& \bigcup_{\theta \in C} j_{C}(\theta)=j(C), \\
& j_{C}\left(\theta_{i}\right) \cap j_{C}\left(\theta_{j}\right)=\emptyset, \quad i \neq j, \\
& j_{C}(\theta)=\emptyset \text { if } \theta \in A, C \cap A \neq \emptyset \text { and } C \nsubseteq A .
\end{aligned}
$$

Let $i(\{\theta\})=\bigcup_{\theta \in C, C \subseteq \Theta} j_{C}(\theta)$ and $i(B)=\bigcup_{\theta \in B} i(\{\theta\})$. Clearly, $i$ satisfies (A5) and (A9), and hence it is a non-numeric probability. Suppose $w \in \underline{i}(B)$, where $B$ is any 
subset of $\Theta$. There exists a subset $C \subseteq B$ such that $w \in j(C)$. By the construction of $j_{C}$, there exists a $\theta \in C$ such that $w \in j_{C}(\theta)$. By the definition of $i$, it follows $w \in i(\{\theta\}) \subseteq i(B)$. Thus, $\underline{i}(B) \subseteq i(B)$. Similarly, we can show that for any $B \subseteq \Theta$, $i(B) \subseteq \bar{i}(B)$. That is, for all $B \subseteq \Theta, \underline{i}(B) \subseteq i(B) \subseteq \bar{i}(B)$, namly, $i \in \mathcal{I}$. Now suppose $w \in i(A)$. There exists a $\theta \in A$ such that $w \in i(\{\theta\})=\bigcup_{\theta \in C, C \subseteq \Theta} j_{C}(\theta)$. By the construction of $j_{C}$, this implies that there exists a set $C \subseteq \Theta$ such that $\theta \in C$ and $w \in j_{C}(\theta)$. Combining these facts with property (D3), we can conclude that $C \subseteq A$. By definition, $w \in j(C)$ and $w \in \underline{i}(A)$. Thus, $i(A)=\underline{i}(A)$. Therefore, $\underline{i}(A)=\inf _{i \in \mathcal{I}} i(A)$. We can show that $\bar{i}$ is the upper envelope of $\mathcal{I}$ in a similar manner.

The notion of non-numeric probability has been studied in various contexts because of its truth functionality with respect to both $\cap$ and $\cup$. The translation mapping proposed by Luzeaux [1991] for symbolic reasoning is essentially a non-numeric probability function. Orlowska [1985] used the same idea to establish a basis for analyzing vague concepts. Similar notions were also used in the studies of possible-worlds semantics for probabilistic reasoning [Fagin and Halpern, 1991; Nilsson, 1986; Ruspini, 1991a].

\subsection{Necessity and Possibility}

Another class of non-numeric belief functions is characterized by axioms (A1), (A2), (A4) and (A6). Its dual $i^{\prime}$ is defined by axioms (A1), (A2), (A5) and (A6). According to axioms (A4) and (A6), $i(A \cap B)$ is the smaller set of $i(A)$ and $i(B)$; according to axioms (A5) and (A6), $i^{\prime}(A \cup B)$ is the larger set of $i^{\prime}(A)$ and $i^{\prime}(B)$. The corresponding numeric measure $f$ is a necessity measure obeying axioms (F1), (F2) and

$$
f(A \cap B)=\min \{f(A), f(B)\} .
$$

The dual $f^{\prime}$, corresponding to $i^{\prime}$, is a possibility measure defined by axioms (F1), (F2) and

$$
f^{\prime}(A \cup B)=\max \left\{f^{\prime}(A), f^{\prime}(B)\right\} .
$$

We may therefore call a non-numeric function satisfying axioms (A1), (A2), (A4) and (A6) a non-numeric necessity function and its dual a non-numeric possibility function. 
Necessity and possibility measures are also called consonant belief and plausibility measures [Dubois and Prade, 1986, 1989; Klir and Folger, 1988; Shafer, 1976; Smets, 1988]. The focal elements of a consonant belief measure are nested. That is, one can arrange the focal elements in such a way that $A_{1} \subset A_{2} \subset \ldots \subset A_{m}$. A possibility measure can be defined by a possibility distribution $\mu: \Theta \longrightarrow[0,1]$ with $\mu(\theta)=$ $f^{\prime}(\{\theta\})$. The possibility of any proposition $A \subseteq \Theta$ is given by:

$$
f^{\prime}(A)=\max _{\theta \in A} \mu(\theta)
$$

Likewise, the focal sets of a non-numeric necessity function form a nested sequence of subsets of $\Theta$. A non-numeric possibility function can be defined by a non-numeric possibility distribution $h: \Theta \longrightarrow 2^{W}$ with $h(\theta)=i^{\prime}(\{\theta\})$. According to axioms (A3) and (A5), the non-numeric possibility of a proposition $A$ is the largest set of $h(\theta)$, where $\theta \in A$.

\subsection{Summary}

The relationships between different classes of non-numeric functions can be summarized as follows. A non-numeric necessity/possibility function is a non-numeric belief/plausibility function. A non-numeric probability function is both a non-numeric belief and plausibility function. A non-numeric belief/plausibility function is a nonnumeric fuzzy measure. Similar relationships also hold for the corresponding classes of numeric measures [Wong, Yao and Lingras, 1993].

In the above discussion, using the normalized cardinality function $f$ defined by equation (1), we have examined the correspondence between non-numeric functions and commonly used numeric measures of uncertainty. For this purpose, one may in fact use a probability measure instead. With a probability measure on $W$, we can define a numeric measure by:

$$
f(A)=P(i(A))
$$

It is not difficult to see that the previous conclusions remain valid if equation (11) is used instead of equation (1) in the above analysis. Thus, non-numeric functions can be used to study the connection between a probability measure and other classes of 
measures of uncertainty. For example, one can infer, from a probability measure on a frame $W$, a belief measure on another frame $\Theta$ through a non-numeric belief function [Corrêa da Silva and Bundy, 1991; Wong, Wang and Yao, 1992b]. One can draw similar conclusions by analyzing other classes of non-numeric functions, as indicated by following theorem.

THEOREM 3 Suppose $P$ is a probability measure on $W$. A function defined by $f(A)=P(i(A))$ is a fuzzy, a belief/plausibility, a probability, or a necessity/possibility measure if $i$ is a non-numeric fuzzy, a non-numeric belief/plausibility, a non-numeric probability, or a non-numeric necessity/possibility function.

This theorem can be easily proved by following the previous discussion. Since the function $|i(A)| /|W|$ is a probability measure, Theorem 3 thus summarizes the main results of this section, and establishes an important linkage between numeric measures and non-numeric functions. As will be shown in the next section, many applications of non-numeric functions stem from this theorem.

The non-numeric axioms introduced in this study are primarily for drawing the correspondence between numeric measures of uncertainty and non-numeric functions. However, these axioms require further investigation. For instance, axiom (A6) seems to be too stringent an axiom to be imposed on a non-numeric function, although it models the corresponding axioms of necessity and possibility measures. Axioms (A4) and (A6) are only sufficient conditions for $f$ to be a necessity measure. It is not clear if they are necessary conditions. If they are not, weaker axioms should perhaps be used.

\section{REASONING WITH NON-NUMERIC FUNC- TIONS}

Non-numeric functions can be defined over any two sets. The applications of these functions depend of course on the meaning of the sets involved and the type of nonnumeric functions used in a particular situation. This section will address some of these issues in the context of uncertain reasoning. 


\subsection{Non-numeric Functions Induced by a Compatibility Relation}

Given two sets $W$ and $\Theta$, the relationship between their elements can be modeled by a compatibility relation [Shafer, 1987]. A compatibility relation is defined as a subset of pairs $(w, \theta)$ in the Cartesian product $W \times \Theta$. An element $w \in W$ is compatible with an element $\theta \in \Theta$, written $w \mathcal{C} \theta$, if $w$ is related to $\theta$. The interpretation of $W$, $\Theta$ and the compatibility relation between these two sets depend on the knowledge at hand and the application itself. For example, in a medical diagnosis system, $W$ may be a set of symptoms and $\Theta$ a set of diseases. A symptom $w$ is said to be compatible with a disease $\theta$ if the symptom $w$ does not rule out the possibility that the patient may have contracted the disease $\theta$. Without loss of generality, we may assume for any $w \in W$ there exists a $\theta \in \Theta$ such that $w \mathcal{C} \theta$, and vice versa.

A compatibility relation $\mathcal{C}$ between $W$ and $\Theta$ can be conveniently described by a multi-valued mapping $\gamma: W \longrightarrow 2^{\Theta}$ [Dempster, 1967; Shafer, 1987]:

$$
\gamma(w)=\{\theta \in \Theta \mid w \mathcal{C} \theta\}
$$

By using $\gamma$, let us define two non-numeric functions from $2^{\Theta}$ to $2^{W}$ :

$$
\underline{i}(A)=\{w \in W \mid \gamma(w) \subseteq A\}
$$

and

$$
\bar{i}(A)=\{w \in W \mid \gamma(w) \cap A \neq \emptyset\} .
$$

For an arbitrary subset $A \subseteq \Theta$, the set $\underline{i}(A)$ consists of all the elements in $W$ compatible with only those elements in $A$, while the set $\bar{i}(A)$ consists of all the elements in $W$ compatible with at least one element in $A$.

Clearly, both mappings $\underline{i}$ and $\bar{i}$ satisfy axioms (A1) and (A2). It can be easily verified that $\underline{i}$ satisfies axiom (A4), $\bar{i}$ satisfies (A5), and for any $A \subseteq \Theta, \bar{i}(A)=$ $W-\underline{i}\left(A^{c}\right)$ [Pawlak, 1982; Shafer, 1976]. Thus, a multi-valued mapping induces a pair of non-numeric belief and plausibility functions. The corresponding basic set assignment is defined by:

$$
j(A)=\{w \mid \gamma(w)=A\}
$$


That is, $j(A)$ consists of all those $w$ 's which are compatible with every element in $A$ but not compatible with any element outside $A$.

Consider now a special type of compatibility relation in which an element of $W$ is compatible with exactly one element of $\Theta$. In this case, the multi-valued mapping becomes a single-valued mapping. The two non-numeric functions defined by equations (13) and (14) reduce to the same function, i.e., $\underline{i}(A)=\bar{i}(A)$ for all $A \subseteq \Theta$. Moreover, this function satisfies both axioms (A4) and (A5). That is, a single-valued mapping induces a non-numeric probability function. It can be seen from equations (12) and (15) that all the focal sets are singleton sets.

Another type of compatible relation can be characterized by the condition:

$$
\gamma\left(w_{i}\right) \subseteq \gamma\left(w_{j}\right) \text { or } \gamma\left(w_{j}\right) \subseteq \gamma\left(w_{i}\right), \quad \text { for } \quad w_{i}, w_{j} \in W
$$

This means that for any two elements $w_{i}$ and $w_{j}$, either $w_{i}$ is compatible with every element of $\Theta$ that is compatible with $w_{j}$, or the reverse relationship holds. The elements of $W$ can be ordered such that $\gamma\left(w_{1}^{\prime}\right) \subseteq \gamma\left(w_{2}^{\prime}\right) \subseteq \ldots, \subseteq \gamma\left(w_{m}^{\prime}\right)$, where $m$ is the cardinality of $W$. By combining equations (15) and (16), we can see that the focal sets are nested. The non-numeric function $\underline{i}$ satisfies axioms (A1), (A2), (A4) and (A6), while $\bar{i}$ satisfies axioms (A1), (A2), (A5) and (A6). In short, under condition (16), a compatibility relation induces a pair of non-numeric necessity and possibility functions.

It is impossible, however, to construct a non-numeric fuzzy measure directly from a compatibility relation between $W$ and $\Theta$. One plausible generalization is to consider a compatibility relation between the subsets of $W$ and the elements of $\Theta$. In other words, one may use a generalized multi-valued mapping $\Gamma: 2^{W} \longrightarrow 2^{\Theta}$. This mapping is more flexible in expressing the relationship between the elements of two sets. For example, a physician may feel uncomfortable in expressing the compatibility between the individual symptoms and diseases, but is able to specify the compatibility between a group of symptoms and a particular disease. We may assume that $\Gamma(X)=\emptyset$ if $X$ is not compatible with any element of $\Theta$, and $\bigcup_{X \subseteq W} \Gamma(X)=\Theta$.

Based on such a mapping $\Gamma$, a pair of dual non-numeric functions may be defined 
as follows:

$$
\underline{i}(A)=\bigcup_{\substack{\emptyset \neq X \subseteq W \\ \Gamma(X) \subseteq A}} X
$$

and

$$
\bar{i}(A)=\bigcup_{\substack{\emptyset \neq X \subseteq W \\ \Gamma(X) \cap A \neq \emptyset}} X
$$

By assumption, $\underline{i}(\emptyset)=\bar{i}(\emptyset)=\emptyset$ and $\underline{i}(\Theta)=\bar{i}(\Theta)=W$. These two non-numeric functions also satisfy axioms (A3), which are indeed a pair of dual non-numeric fuzzy measures.

Example 1 This simple example illustrates the process of constructing various nonnumeric functions from compatibility relations. Let $W=\left\{w_{1}, w_{2}, w_{3}, w_{4}\right\}$ and $\Theta=$ $\left\{\theta_{1}, \theta_{2}, \theta_{3}\right\}$. Consider the following compatibility relations:

$$
\begin{array}{llll}
\gamma_{1}\left(w_{1}\right)=\left\{\theta_{1}, \theta_{2}\right\}, & \gamma_{1}\left(w_{2}\right)=\left\{\theta_{2}, \theta_{3}\right\}, & \gamma_{1}\left(w_{3}\right)=\left\{\theta_{3}\right\}, & \gamma_{1}\left(w_{4}\right)=\left\{\theta_{1}, \theta_{3}\right\} \\
\gamma_{2}\left(w_{1}\right)=\left\{\theta_{1}\right\}, & \gamma_{2}\left(w_{2}\right)=\left\{\theta_{2}\right\}, & \gamma_{2}\left(w_{3}\right)=\left\{\theta_{3}\right\}, & \gamma_{2}\left(w_{4}\right)=\left\{\theta_{3}\right\} \\
\gamma_{3}\left(w_{1}\right)=\left\{\theta_{1}\right\}, & \gamma_{3}\left(w_{2}\right)=\left\{\theta_{1}\right\}, & \gamma_{3}\left(w_{3}\right)=\left\{\theta_{1}, \theta_{2}\right\}, & \gamma_{3}\left(w_{4}\right)=\left\{\theta_{1}, \theta_{2}, \theta_{3}\right\}
\end{array}
$$

The induced non-numeric functions for $\gamma_{1}$ and $\gamma_{2}$ are summarized in Table 1. Note that $\underline{i}_{1}$ and $\bar{i}_{1}$ define a pair of non-numeric belief and plausibility functions, while $i_{2}=\underline{i}_{2}=\bar{i}_{2}$ defines a non-numeric probability function. The basic set assignment $j_{2}$ of $i_{2}$ may be viewed as a non-numeric probability distribution over $W$. The nonnumeric probability function $i_{2}$ is bounded by $\underline{i}_{1}$ and $\bar{i}_{1}$.

Table 1. Examples of non-numeric belief, plausibility and probability functions induced by compatibility relations.

\begin{tabular}{|c|ccc|cc|}
\hline$A$ & $j_{1}$ & $\underline{i}_{1}$ & $\bar{i}_{1}$ & $j_{2}$ & $i_{2}=\underline{i}_{2}=\bar{i}_{2}$ \\
\hline$\emptyset$ & $\emptyset$ & $\emptyset$ & $\emptyset$ & $\emptyset$ & $\emptyset$ \\
$\left\{\theta_{1}\right\}$ & $\emptyset$ & $\emptyset$ & $\left\{w_{1}, w_{4}\right\}$ & $\left\{w_{1}\right\}$ & $\left\{w_{1}\right\}$ \\
$\left\{\theta_{2}\right\}$ & $\emptyset$ & $\emptyset$ & $\left\{w_{1}, w_{2}\right\}$ & $\left\{w_{2}\right\}$ & $\left\{w_{2}\right\}$ \\
$\left\{\theta_{3}\right\}$ & $\left\{w_{3}\right\}$ & $\left\{w_{3}\right\}$ & $\left\{w_{2}, w_{3}, w_{4}\right\}$ & $\left\{w_{3}, w_{4}\right\}$ & $\left\{w_{3}, w_{4}\right\}$ \\
$\left\{\theta_{1}, \theta_{2}\right\}$ & $\left\{w_{1}\right\}$ & $\left\{w_{1}\right\}$ & $\left\{w_{1}, w_{2}, w_{4}\right\}$ & $\emptyset$ & $\left\{w_{1}, w_{2}\right\}$ \\
$\left\{\theta_{1}, \theta_{3}\right\}$ & $\left\{w_{4}\right\}$ & $\left\{w_{3}, w_{4}\right\}$ & $W$ & $\emptyset$ & $\left\{w_{1}, w_{3}, w_{4}\right\}$ \\
$\left\{\theta_{2}, \theta_{3}\right\}$ & $\left\{w_{2}\right\}$ & $\left\{w_{2}, w_{3}\right\}$ & $W$ & $\emptyset$ & $\left\{w_{2}, w_{3}, w_{4}\right\}$ \\
$\Theta$ & $\emptyset$ & $W$ & $W$ & $\emptyset$ & $W$ \\
\hline
\end{tabular}


The multi-valued mapping $\gamma_{3}$, with $\gamma_{3}\left(w_{1}\right) \subseteq \gamma_{3}\left(w_{2}\right) \subseteq \gamma_{3}\left(w_{3}\right) \subseteq \gamma_{3}\left(w_{4}\right)$, induces a pair of non-numeric necessity and possibility functions $\underline{i_{3}}$ and $\overline{i_{3}}$. The corresponding non-numeric possibility distribution is denoted by $h$. These mappings are shown in Table 2 .

Table 2. An example of non-numeric necessity and possibility functions induced by a compatibility relation.

\begin{tabular}{|c|cccc|}
\hline$A$ & $j_{3}$ & $h$ & $\underline{i}_{3}$ & $\bar{i}_{3}$ \\
\hline$\emptyset$ & $\emptyset$ & - & $\emptyset$ & $\emptyset$ \\
$\left\{\theta_{1}\right\}$ & $\left\{w_{1}, w_{2}\right\}$ & $W$ & $\left\{w_{1}, w_{2}\right\}$ & $W$ \\
$\left\{\theta_{2}\right\}$ & $\emptyset$ & $\left\{w_{3}, w_{4}\right\}$ & $\emptyset$ & $\left\{w_{3}, w_{4}\right\}$ \\
$\left\{\theta_{3}\right\}$ & $\emptyset$ & $\left\{w_{4}\right\}$ & $\emptyset$ & $\left\{w_{4}\right\}$ \\
$\left\{\theta_{1}, \theta_{2}\right\}$ & $\left\{w_{3}\right\}$ & - & $\left\{w_{1}, w_{2}, w_{3}\right\}$ & $W$ \\
$\left\{\theta_{1}, \theta_{3}\right\}$ & $\emptyset$ & - & $\left\{w_{1}, w_{2},\right\}$ & $W$ \\
$\left\{\theta_{2}, \theta_{3}\right\}$ & $\emptyset$ & - & $\emptyset$ & $\left\{w_{3}, w_{4}\right\}$ \\
$\Theta$ & $\left\{w_{4}\right\}$ & - & $W$ & $W$ \\
\hline
\end{tabular}

Consider now a generalized multi-valued mapping:

$$
\begin{aligned}
& \Gamma\left(\left\{w_{1}, w_{2}\right\}\right)=\left\{\theta_{1}, \theta_{2}\right\}, \quad \Gamma\left(\left\{w_{2}, w_{3}, w_{4}\right\}\right)=\left\{\theta_{2}, \theta_{3}\right\}, \\
& \Gamma(X)=\emptyset, \text { for all other subsets of } W .
\end{aligned}
$$

The induced non-numeric functions are given in Table 3 . The mappings $\underline{i}$ and $\bar{i}$ define a pair of dual non-numeric fuzzy measures.

Table 3. An example of non-numeric fuzzy measures induced by a compatibility relation. 


\begin{tabular}{|c|cc|}
\hline$A$ & $\underline{i}$ & $\bar{i}$ \\
\hline$\emptyset$ & $\emptyset$ & $\emptyset$ \\
$\left\{\theta_{1}\right\}$ & $\emptyset$ & $\left\{w_{1}, w_{2}\right\}$ \\
$\left\{\theta_{2}\right\}$ & $\emptyset$ & $W$ \\
$\left\{\theta_{3}\right\}$ & $\emptyset$ & $\left\{w_{2}, w_{3}, w_{4}\right\}$ \\
$\left\{\theta_{1}, \theta_{2}\right\}$ & $\left\{w_{1}, w_{2}\right\}$ & $W$ \\
$\left\{\theta_{1}, \theta_{3}\right\}$ & $\emptyset$ & $W$ \\
$\left\{\theta_{2}, \theta_{3}\right\}$ & $\left\{w_{2}, w_{3}, w_{4}\right\}$ & $W$ \\
$\Theta$ & $W$ & $W$ \\
\hline \multicolumn{2}{|c}{} \\
\hline
\end{tabular}

\subsection{Non-numeric Functions and Possible-worlds Semantics}

This section provides another example to demonstrate the usefulness of non-numeric functions for uncertain reasoning.

One salient feature of our approach is that the requirement of truth functionality is not necessary for a possible world assignment. This distinguishes our analysis from the studies of Corrêa da Silva and Bundy [1990], Fagin and Halpern [1991], Nilsson [1986], and Ruspini [1991a, 1991b].

Let $\Phi$ denote a finite non-empty set of propositions. A propositional language formed from $\Phi$ is denoted by $L(\Phi)$, which is the smallest set containing the truth values and the members of $\Phi$. This set is closed under negation $(\neg)$ and conjunction $(\wedge)$. Other connectives such as the disjunction $(\vee)$, implication $(\rightarrow)$ and equivalence $(\leftrightarrow)$ can be defined in terms of negation and conjunction. We assume that there is a special formula $T$. Its negation $\neg T$ is written as $F$. Given the set of finite basic propositions $\Phi=\left\{p_{1}, \ldots, p_{n}\right\}$, we consider the set $A t$ of all the atoms of the form $p_{1}^{\prime} \wedge \ldots \wedge p_{n}^{\prime}$, where $p_{i}^{\prime}$ is either $p_{i}$ or $\neg p_{i}$. Any formula $\phi \in L(\Phi)$ can be expressed as a disjunctive normal form, i.e., a disjunction of atoms. Consequently, any formula $\phi$ is uniquely described by a subset $\left\{\delta_{1}, \ldots, \delta_{k}\right\} \subseteq A t$ such that $\phi=\delta_{1} \vee \ldots \vee \delta_{k}$. The power set $2^{A t}$ represents all the formulas constructed from $\Phi$, with $\emptyset$ for $F$ and At for $T$. In the following discussion, propositions and subsets of $A t$ will be used interchangeably.

Let $W$ be a non-empty set of possible worlds representing states or situations of the system being modeled. Each possible world provides partial information about 
some logical formula in $L(\Phi)$. For a particular possible world $w$, a total valuation function $\pi_{w}: \Phi \longrightarrow\{$ true, false $\}$ may be defined [Fagin and Halpern, 1991; Ruspini, 1991b]. If $\pi_{w}(\phi)=$ true, the proposition $\phi$ is said to be true in the world $w$; otherwise it is false. According to the semantics of two-valued logic, given two propositions $\phi$ and $\psi, \phi \wedge \psi$ is true in a world $w$, if and only if both $\phi$ and $\psi$ are true in $w$. Similar rules can be used for other operations. Thus, the valuation function $\pi_{w}$ can be extended to $L(\Phi)$, or more conveniently to $2^{A t}$. Alternatively, one can define the truth assignment for all atoms. The valuation of other propositions can be deduced using the truth functionality of the propositional logic [Fagin and Halpern, 1991; Nilsson, 1986].

From the valuation functions $\pi_{w}$ for all possible worlds, a mapping $i: 2^{A t} \longrightarrow 2^{W}$ can be defined as follows:

$$
i(\phi)=\left\{w \in W \mid \pi_{w}(\phi)=\text { true }\right\}
$$

That is, the set $i(\phi)$ contains those possible worlds in which $\phi$ is true. It is interpreted as the incidence set or value of the proposition [Bundy, 1985, 1986]. The proposition $\emptyset$ is false in every possible world, while the proposition $A t$ is true in every possible world. In fact, the non-numeric function (19) satisfies axioms (A4) and (A5). Thus, it defines a non-numeric probability. This provides the possible-worlds semantics for probabilistic reasoning. If a numeric probability measure is defined on $W$, then the probability of a proposition is defined by the probability of its incidence set [Fagin and Halpern, 1991; Nilsson, 1986].

In many situations, it may be more suitable to use a partial valuation function, rather than a total function [Ruspini, 1991a; Smets, 1988]. For a particular possible world, three possible states are allowed: the proposition is known to be true, the proposition is known to be false, or the available information may be insufficient to determine the truth or falsity of the proposition. In this case, it is impossible to apply the truth functionality of propositional logic. A valuation function must be given for every proposition in $2^{A t}$. Given a possible world $w \in W$, a partial valuation function, $\pi_{w}: 2^{A t} \longrightarrow\{$ true, false $\}$ can be defined. By $\pi_{w}(\phi)=$ true, we mean that the proposition $\phi$ is known or proved to be true in the world $w$, and $\pi_{w}(\phi)=$ false 
otherwise. With this interpretation, the following condition should be obeyed:

$$
\left[\pi_{w}(\phi)=\text { true }\right] \Longleftrightarrow\left[\pi_{w}(\neg \phi)=\text { false }\right]
$$

For clarity, it is assumed that $\pi_{w}(\emptyset)=$ false and $\pi_{w}(A t)=$ true for every possible world $w \in W$.

With partial valuation functions, two dual non-numeric functions, $\underline{i}: 2^{A t} \longrightarrow 2^{W}$ and $\bar{i}: 2^{A t} \longrightarrow 2^{W}$, can be defined as follows:

$$
\begin{aligned}
& \underline{i}(\phi)=\left\{w \in W \mid \pi_{w}(\phi)=\text { true }\right\}, \\
& \bar{i}(\phi)=W-\underline{i}(\neg \phi) .
\end{aligned}
$$

Clearly, when partial evaluations become total valuation functions, the non-numeric functions $\underline{i}$ and $\bar{i}$ reduce to the same non-numeric probability. In general, we call $\underline{i}(\phi)$ the lower incidence set, and $\bar{i}(\phi)$ the upper incidence set, of $\phi$. Other types of nonnumeric functions can be defined depending on the properties obeyed by the partial valuation functions.

Suppose the truth functionality of $\wedge$ is provided by a partial mapping, while the truth functionality of $\vee$ is not. Based on the interpretation of possible-worlds semantics, the partial valuation should satisfy the following conditions:

$$
\begin{aligned}
& {\left[\pi_{w}(\phi)=\text { true and } \pi_{w}(\psi)=\text { true }\right] \Longleftrightarrow\left[\pi_{w}(\phi \wedge \psi)=\text { true }\right]} \\
& {\left[\pi_{w}(\phi)=\text { true or } \pi_{w}(\psi)=\text { true }\right] \Longrightarrow\left[\pi_{w}(\phi \vee \psi)=\text { true }\right]}
\end{aligned}
$$

In fact, condition (22) implies condition (23). In this case, one may say that $\pi_{w}(\phi \vee$ $\psi)=$ true without stating $\pi_{w}(\phi)=$ true or $\pi_{w}(\psi)=$ true. In other words, one may commit to a composite proposition without making any commitment to its components. This is similar to the construction of belief measures, in which one may assign belief to a set without distributing such belief to its subsets [Shafer, 1976]. According to definition (21), $\underline{i}$ satisfies axiom (A4), while $\bar{i}$ satisfies axiom (A5). They define a pair of non-numeric belief and plausibility functions. The set $\bar{i}(\phi)-\underline{i}(\psi)$ denotes the set of possible worlds in which the truth value of $\phi$ is undeterminable. This notion is similar to the doubtful region in the rough-set theory [Pawlak, 1982]. 
From Theorem 3, one can conclude that if a numeric probability measure is defined on $W$, the belief of a proposition can be defined by the probability of its lower incidence set, while the plausibility by the probability of its upper incidence set. The notion of non-numeric belief functions therefore provides the possible-worlds semantics for reasoning with numeric belief/plausibility measures.

In addition to condition (22), assume that possible worlds can be arranged into a sequence $w_{1}, \ldots, w_{m}$ such that for any proposition $\phi$,

$$
\left(\pi_{w_{i}}(\phi)=\text { true }\right) \Longrightarrow\left(\pi_{w_{j}}(\phi)=\text { true }\right), \quad \text { for any } i, j \text { with } i<j
$$

Under these conditions, the non-numeric function $\underline{i}$ satisfies axioms (A4) and (A6), and $\bar{i}$ satisfies axioms (A5) and (A6). They form a pair of non-numeric necessity and possibility functions. Based on Theorem 3, this provides possible-worlds semantics for possibilistic logic.

Now suppose the truth functionality is not provided for both $\wedge$ and $\vee$ in specifying the partial valuation function. Using the following condition:

$$
(\phi \rightarrow \psi) \Longrightarrow\left[\left(\pi_{w}(\phi)=\text { true }\right) \Longrightarrow\left(\pi_{w}(\psi)=\text { true }\right)\right]
$$

a pair of dual non-numeric fuzzy measures can be derived, which provides the possibleworlds semantics for reasoning with fuzzy measures.

\section{CONCLUSION}

In this paper, we have presented an analysis on different classes of non-numeric functions and their applications in uncertain reasoning. The fundamental issues being examined include the characterization, classification, construction and interpretation of a number of non-numeric functions. With respect to the well known fuzzy, belief/plausibility, probability, and necessity/possibility measures, we have analyzed the corresponding classes of non-numeric functions. For each class, we have studied the relationships between the qualitative and quantitative axioms, and analyzed the properties of the non-numeric and numeric measures. We have also discussed how to construct a non-numeric function from a compatibility relation. In fact, non-numeric 
functions can be adopted to provide the possible-worlds semantics for uncertain reasoning. Thus, this preliminary investigation establishes a basis for using non-numeric functions to represent uncertainty.

Resconi, Klir and Clair [1992], Resconi et. al. [1993], and Harmanec, Klir and Resconi [1993] proposed a unifying framework for the study of various uncertainty theories. The present investigation is closely related to their work. Both methods use a similar technique to relate non-numeric functions and numeric measures. However, we used non-numeric functions as the primitive concept, while they used modal logic as the underlying notion. It will be useful to combine these two frameworks by relating the axioms of non-numeric functions to the axioms of modal logic operators. Yao and $\mathrm{Li}$ [1993] adopted Kleene's three-valued logic in which an additional truth value is used [Rescher, 1969]. Their work is intended to provide a possible-worlds semantics for interval-based probabilistic reasoning. It would be interesting to extend our work to investigate other interval-based methods. The relationship between distinct possible worlds was used by Ruspini [1991b] to develop the possible-worlds semantics for fuzzy logic. Such a relationship can be included into the present framework.

\section{ACKNOWLEDGEMENT}

The authors wish to thank the reviewers of this paper for their valuable comments and helpful suggestions.

\section{REFERENCES}

Bhatnagar, R.K. and L.N. Kanal [1986], "Handling uncertain information: a review of numeric and non-numeric methods." In: Uncertainty in Artificial Intelligence, edited by L.N. Kanal and J.F. Lemmer, North-Holland, New York, pp. $3-26$.

Bundy, A. [1985], "Incidence calculus: a mechanism for probabilistic reasoning." Journal of Automated Reasoning, 1, pp. 263-283.

Bundy, A. [1986], "Correctness criteria of some algorithms for uncertain reasoning using incidence calculus." Journal of Automated Reasoning, 2, pp. 109-126. 
Corrêa da Silva, F. and A. Bundy [1990], "On some equivalence relations between incidence calculus and Dempster-Shafer theory of evidence." Proceedings of the Sixth International Workshop on Uncertainty in Artificial Intelligence, pp. 378-383.

Dempster, A.P. [1967], "Upper and lower probabilities induced by a multivalued mapping." Annals of Mathematical Statistics, 38, pp. 325-339.

Dubois, D. and H. Prade [1986], "A set-theoretic view of belief functions: logical operations and approximation by fuzzy sets." International Journal of General Systems, 12, pp. 193-226.

Dubois, D. and H. Prade [1988], "An introduction to possibilistic and fuzzy logics (with discussions)." In: Non-standard Logics for Automated Reasoning, edited by P. Smets, A. Mamdani, D. Dubois and H. Prade, Academic Press, New York, pp. 287-386.

Dubois, D. and H. Prade [1989], "Fuzzy sets, probability and measurement." European Journal of Operational Research, 40, pp. 135-154.

Fagin, R. and J.Y. Halpern [1991], "Uncertainty, belief, and probability." Computational Intelligence, 6, pp. 160-173.

Harmanec, D., G.J. Klir and G. Resconi [1993], "Completing interpretation of Dempster-Shafer theory within the semantics of modal logic." Proceedings of the Second European Congress on Systems Science, pp. 1031-1038.

Jean-Louis, R. [1991], "Topological characterization of common knowledge: some extensions to the game-theoretic model of the knowledge operator." In: Decision Support Systems and Qualitative Reasoning, edited by M.G. Singh and L. Travé-Massuyès, Elsevier (North-Holland), New York, pp. 127-132.

Klir, G.J. and T.A. Folger [1988], Fuzzy sets, Uncertainty, and Information. Prentice Hall, Englewood Cliffs, New Jersey.

Luzeaux, D. (1991). Formalizing symbolic reasoning. In: Decision Support Systems and Qualitative Reasoning, edited by M.G. Singh and L. Travé-Massuyès, Elsevier (North-Holland), New York, pp. 315-320.

Nguyen, H.T. [1978], "On random sets and belief functions." Journal of Mathematical Analysis and Applications, 65, pp. 531-542. 
Nilsson, N.J. [1986], "Probabilistic logic." Artificial Intelligence, 28, pp. 71-87.

Orlowska, E. [1985], "Semantics of vague concepts." In: Foundations of Logic and Linguistics: Problems and Solutions, edited by G. Dorn and P. Weingartner, Plenum Press, New York, pp. 465-482.

Pawlak, Z. [1982], "Rough sets." International Journal of Computer and Information Sciences, 11, pp. 341-356.

Pawlak, Z. [1984], "Rough classification." International Journal of Man-Machine Studies, 20, pp. 469-483.

Rescher, N. [1969], Many-valued Logic, McGraw-Hill, New York.

Resconi, G., G.J. Klir and U.St. Clair [1992], "Hierarchical uncertainty metatheory based upon modal logic." International Journal of General Systems, 21, pp. 23-50.

Resconi, G., G.J. Klir, U.St. Clair and D. Harmanec [1993], "On the integration of uncertainty theories." International Journal of Uncertainty, Fuzziness and Knowledge-Based Systems, 1, pp. 1-18.

Ruspini, E.H. [1991a], "Approximate reasoning: past, present, future." Information Sciences, 57-58, pp. 297-313.

Ruspini, E.H. [1991b], "On the semantics of fuzzy logic." International Journal of Approximate Reasoning, 5, pp. 45-88.

Shafer, G. [1976], A Mathematical Theory of Evidence, Princeton University Press, Princeton.

Shafer, G. [1987], "Belief functions and possibility measures." In: Analysis of Fuzzy Information, vol. 1: Mathematics and Logic, edited by J.C. Bezdek, CRC Press, Boca Raton, Florida, pp. 51-84.

Shortliffe, E.H. [1976], Computer-Based Medical Consultations: MYCIN, Elsevier (North-Holland), New York.

Smets, P. [1988], "Belief functions (with discussion)." In: Non-Standard Logics for Automated Reasoning, edited by P. Smets, A. Mamdani, D. Dubois, and H. Prade, Academic Press, New York, pp. 282-285. 
Sugeno, M. [1974], Theory of Fuzzy Integral and Its Applications. Ph.D. Thesis, Tokyo Institute of Technology, Tokyo, Japan.

Wong, S.K.M., L.S. Wang, and Y.Y. Yao [1992a], "Interval structures: a framework for representing uncertain information." Proceedings of the Eighth Conference on Uncertainty in Artificial Intelligence, pp. 336-343.

Wong, S.K.M., L.S. Wang, and Y.Y. Yao [1992b], "Non-numeric Belief structures." Proceedings of the Fourth International Conference on Computing and Information, pp. 274-277.

Wong, S.K.M., Y.Y. Yao, and P. Lingras [1993], "Comparative beliefs and their measurements." International Journal of General Systems, 22, pp. 69-90.

Yao, Y.Y. [1993], "Interval-set algebra for qualitative knowledge representation." Proceedings of the Fifth International Conference on Computing and Information, pp. 370-374.

Yao, Y.Y. and X. Li [1993], "Uncertain reasoning with interval-set algebra." Proceedings of the International Workshop on Rough Sets and Knowledge Discovery, first edition, pp. 191-202. 